\title{
Diet quality score is a predictor of type 2 diabetes risk in women: The Australian Longitudinal Study on Women's Health
}

\author{
Amani Alhazmi ${ }^{1}$, Elizabeth Stojanovski ${ }^{2}$, Mark McEvoy ${ }^{3}$, Wendy Brown ${ }^{4}$ and Manohar L. Garg ${ }^{5 *}$ \\ ${ }^{1}$ Discipline of Nutrition and Dietetics, Faculty of Health, School of Health Sciences, University of Newcastle, Callaghan, \\ NSW, Australia \\ ${ }^{2}$ Faculty of Science and Information Technology, School of Mathematical and Physical Sciences, University of Newcastle, \\ Callaghan, NSW, Australia \\ ${ }^{3}$ Faculty of Health, Centre for Clinical Epidemiology and Biostatistics, University of Newcastle, Callaghan, NSW, Australia \\ ${ }^{4}$ School of Human Movement Studies, University of Queensland, Brisbane, QLD, Australia \\ ${ }^{5}$ School of Biomedical Sciences and Pharmacy, 305C Medical Sciences Building, University of Newcastle, Callaghan, \\ NSW 2308, Australia
}

(Submitted 23 September 2013 - Final revision received 12 February 2014 - Accepted 25 February 2014 - First published online 24 July 2014)

\section{Abstract}

The present study aimed to determine the ability of two diet quality scores to predict the incidence of type 2 diabetes in women. The study population comprised a nationally representative sample of 8370 Australian middle-aged (45-50 years) women participating in the ALSWH (Australian Longitudinal Study on Women's Health), who were free of diabetes and completed FFQ at baseline. The associations between the Australian Recommended Food Score (ARFS) and Dietary Guideline Index (DGI) with type 2 diabetes risk were assessed using multiple logistic regression models, adjusting for sociodemographic characteristics, lifestyle factors and energy intake. During 6 years of follow-up, 311 incident cases of type 2 diabetes were reported. The DGI score was inversely associated with type 2 diabetes risk (OR comparing the highest with the lowest quintile of DGI was $0.51 ; 95 \%$ CI $0 \cdot 35,0 \cdot 76 ; P$ for trend $=0.01)$. There was no statistically significant association between the ARFS and type 2 diabetes risk (OR comparing the highest with the lowest quintile of ARFS was 0.99; $95 \%$ CI $0 \cdot 68,1 \cdot 43 ; P$ for trend $=0 \cdot 42$ ). The results of the present prospective study indicate that the DGI score, which assesses compliance with established dietary guidelines, is predictive of type 2 diabetes risk in Australian women. The risk of type 2 diabetes among women in the highest quintile of DGI was approximately $50 \%$ lower than that in women in the lowest quintile. The ARFS was not significantly predictive of type 2 diabetes.

Key words: Diet quality: Type 2 diabetes: Cohort studies: Women

The prevalence of diabetes poses a substantial health problem worldwide, with 285 million adults being affected in 2010 and an expected increase to 439 million adults being reported to occur by $2030^{(1)}$. In Australia, the prevalence of diabetes, mostly type 2 diabetes, among adults (aged 20-79 years) is $7 \cdot 2 \%$ and is expected to increase to $8 \cdot 4 \%$ by $2030^{(1)}$. The beneficial effects of modifying lifestyle factors such as diet have been reported to reduce the incidence of disease ${ }^{(2,3)}$. Dietary patterns have become a common tool for examining the association between diet and health as they consider the influence of diet as a whole and thus may provide insights beyond the role of nutrients and single foods. Associations between dietary patterns and type 2 diabetes risk have been frequently observed and recently reviewed ${ }^{(4)}$.

Diet quality scores have been used to describe dietary patterns in free-living populations, with burgeoning interest in quantifying the associated risk of some health outcomes, including type 2 diabetes. Methods for assessing diet quality are, however, evolving, particularly in terms of assessing the quality and variety of foods in the overall diet. Prospective cohort studies conducted in the USA have shown that high-quality diets are substantially associated with a lower incidence of type 2 diabetes ${ }^{(5,6)}$.

At least two different dietary indices, based on the Dietary Guidelines for Australian Adults ${ }^{(7)}$ and the Australian Guide to Healthy Eating ${ }^{(8)}$, have been developed to assess adherence to national dietary recommendations and optimal eating patterns in Australia. The first is the Dietary Guideline Index (DGI $)^{(9)}$, which uses an approach similar to those of the US dietary indices $^{(10,11)}$. The DGI has been shown to be significantly associated with lower systolic and diastolic blood pressure among men, lower fasting plasma glucose concentrations

Abbreviations: ARFS, Australian Recommended Food Score; DGI, Dietary Guideline Index.

*Corresponding author: M. L. Garg, fax +612 4921 2028, email manohar.garg@newcastle.edu.au 
among men and women, and lower systolic blood pressure and fasting plasma insulin and $2 \mathrm{~h}$ plasma glucose concentrations and greater insulin sensitivity among women. Diet quality has been shown to be inversely associated with abdominal obesity, hypertension and type 2 diabetes among men ${ }^{(12)}$. However, the latter study is limited by its crosssectional design ${ }^{(12)}$. Adherence to a high-quality diet as measured by the DGI has also been shown to be associated with a lower gain in BMI and waist circumference in middle-aged men ${ }^{(13)}$. Furthermore, the DGI has been shown to be associated with overall improvement in diet quality in Australian adults ${ }^{(14)}$. The second index is the Australian Recommended Food Score (ARFS) ${ }^{(15)}$, which has been modified from the Recommended Food Score ${ }^{(16)}$. A higher ARFS has been shown to be associated with self-reported health status and indices of health service usage ${ }^{(15)}$, but to be not significantly predictive of pregnancy status ${ }^{(17)}$ and health service ${ }^{(18)}$.

The ARFS and DGI were developed based on the Dietary Guidelines for Australian Adults ${ }^{(7)}$ and the Australian Guide to Healthy Eating ${ }^{(8)}$ to assess adherence to national dietary recommendations and optimal eating patterns in Australia. The use of both scores for evaluating the same health outcome allows for the direct comparison of the predictive validity and clinical utility of each score. The aim of the present study was to determine the ability of the DGI and ARFS to predict the incidence of type 2 diabetes in a nationally representative sample of middle-aged women participating in the Australian Longitudinal Study on Women's Health (ALSWH).

\section{Methods}

\section{Study population}

The design of the ALSWH has been described previously ${ }^{(19)}$. Briefly, it is a prospective cohort study that investigates factors affecting the health and well-being of women over a 20-year period. A total of three age cohorts of Australian women (younger (18-23 years), middle-aged (45-50 years) and older ( $70-75$ years)) are randomly selected from the national health insurance database (Medicare) that includes all permanent residents of Australia, but with intentional over-representation of women from rural and remote areas. The study collects self-reported information using a mailed survey at 2- to 3-year intervals. The sample of the present study comprised 9101 middle-aged women who completed the third survey (2001), which included a FFQ. The third survey (2001) was completed by $83 \%$ of women who had completed the first survey (1996) and had not died or become too ill to complete further surveys. The nonrespondents included those who did not complete the third survey $(7 \cdot 4 \%)$, withdrew from the study completely $(2 \cdot 8 \%)$ or could not be contacted $(6 \cdot 8 \%)$. Women who reported a daily energy intake $<3347 \mathrm{~kJ}(800 \mathrm{kcal})$ or $>25104 \mathrm{~kJ}$ $(6000 \mathrm{kcal})(n 291)^{(20)}$ or who had a history of diabetes ( $n$ 440) were excluded, leaving 8370 women, whose data were included in the analyses. The study was approved by the University of Newcastle and the University of Queensland Human Research Ethics Committees.

\section{Assessment of dietary intake}

The Dietary Questionnaire for Epidemiological Studies (DQES v2) was used to assess food intake over the previous 12 months $^{(21)}$. The DQES v2 is a computer-scannable FFQ that is developed by the Cancer Council of Victoria, Australia, and is based on NUTTAB95 nutrient composition tables ${ }^{(22)}$. For each food, ten possible responses for frequency of consumption, ranging from 'never' up to 'three or four times per day', were given. Portion photographs of vegetables, potatoes, meat and casserole dishes were used as a guide for participants to calculate a standard portion size. Additional questions were asked about the number of servings and types of fruit, vegetables, breads, dairy products, eggs, fat spreads and sugar consumed ${ }^{(21)}$. The validation of this FFQ has previously been conducted with sixty-three women of childbearing age against $7 \mathrm{~d}$ weighed food records and found to be useful for the assessment of habitual intake in the Australian population ${ }^{(23)}$. The validation study reported less than $10 \%$ variation in mean nutrient intakes for most nutrients $^{(23)}$. Energy-adjusted correlation coefficients for nutrient intakes ranged from $0 \cdot 28$ (vitamin A) to 0.78 (carbohydrate $)^{(23)}$. At the individual level, there were considerable differences in the intakes estimated by the two methods for some nutrients ${ }^{(23)}$.

\section{Measurement of diet quality scores}

Diet quality scores were measured using the DGI and ARFS. The score calculation is based on regular consumption of FFQ items that align with both the Dietary Guidelines for Australian Adults ${ }^{(7)}$ and the Australian Guide to Healthy Eating $^{(8)}$. The ARFS was developed by Collins et al. $^{(15)}$, according to the method of Kant \& Thompson ${ }^{(16)}$ in the USA, and was computed based on DQES items consistent with national recommendations ${ }^{(8,7)}$. One point was assigned for consumption of any of the recommended food items once or more weekly and zero points were given if consumed less often. One point was assigned for specific types and amounts of core foods consumed including the following: at least two fruit servings daily; at least four vegetable servings daily; high-fibre, wholemeal, rye or multigrain breads; at least four slices of bread daily; polyunsaturated or monounsaturated spreads or no fat spread; one or two eggs weekly; ricotta or cottage cheese; low-fat cheese. If alcohol was consumed, a maximum of two points were given for alcohol intake: one point for moderate frequency and another point for moderate quantity. The maximum ARFS is 74 , derived from one point each for vegetables (twenty-two possible points); fruit (fourteen possible points); grains (fourteen possible points); eggs, nuts, beans, or soya (seven possible points); meat or poultry (five possible points); fish (two possible points); dairy products (seven possible points); fat (one possible point) and alcohol (two possible points) ${ }^{(15)}$.

The DGI was developed by McNaughton et al. ${ }^{(9)}$, according to a method similar to that used for the Healthy Eating Index ${ }^{(10)}$ and the Revised Diet Quality Index ${ }^{(11)}$. It consists of fifteen food components, including dietary indicators of 
vegetables and legumes, fruit, total cereals, meat and alternatives, total dairy products, fluids, salt, saturated fat, alcoholic beverages, added sugars and 'extra food', which was defined as foods that are not essential to meet nutritional requirements and contain excessive amounts of fat, sugar and salt ${ }^{(8)}$. Salt use and fluid intake were excluded from the present analysis due to lack of appropriate measures of these items in the FFQ, leaving thirteen components for consideration in the present study. Each component contributed $0-10$ points, where 10 indicated an optimal diet intake or meeting the recommendation. For example, 10 points were allocated for consuming two servings of fruit per day (recommended amount), 5 points for consuming one serving of fruit per day and 0 points for not consuming fruit ${ }^{(9)}$.

The total DGI score was the sum of the thirteen items, indicating that a maximum possible score range was $0-130$.

\section{Ascertainment of type 2 diabetes}

The presence of diabetes was self-reported. During the third, fourth and fifth surveys, women were asked whether they had been diagnosed with diabetes in the past 3 years, which corresponds to the interval since the previous survey. Diabetes was differentiated into type 1 or type 2 during the third survey when all prevalent cases of either were excluded, but diabetes was not differentiated during the fourth and fifth surveys. However, type 1 diabetes was unlikely to exist during the fourth and fifth surveys, given that cases were excluded in the third survey. The incidence of type 2 diabetes was determined by new cases of diabetes during the fourth and fifth surveys. In a sample of 6921 middle-aged women who completed the fourth survey, $70 \%$ of self-reported cases of type 2 diabetes were confirmed by linkage of data to Medicare (MBS) and the Pharmaceutical Benefits Scheme (PBS) ${ }^{(24)}$

\section{Assessment of covariates}

Items measuring other factors that are potentially associated with diabetes risk were included in the questionnaire. These included area of residence, which was categorised as urban (capital city or other metropolitan centres), rural (large rural centre, small rural centre or other rural) or remote ${ }^{(25)}$, and education, which was categorised as less than year 10 or equivalent (schooling to the age of 15 or 16 years), year 12 or equivalent (schooling to the age of 17 or 18 years), trade/ certificate, or university degree. Physical activity scores were derived from self-reported frequency and intensity of leisure-time physical activity items. The questions were modified slightly from those developed for monitoring and evaluating the national active Australia campaign ${ }^{(26)}$; physical activity was categorised as none, low, moderate or high. Cigarette smoking status was defined as never smoked, ex-smoker, or smoke $<10$ cigarettes/d, 10-19 cigarettes/d or $\geq 20$ cigarettes/d. Menopausal status was classified as postmenopausal, peri-menopausal, premenopausal, surgical menopausal, hormone replacement therapy use or oral contraceptive pill use. BMI was calculated as weight $(\mathrm{kg})$ divided by height $(\mathrm{m})$ squared and categorised according to the recommendations of the WHO: underweight $\left(<18.5 \mathrm{~kg} / \mathrm{m}^{2}\right)$, acceptable weight ( $\geq 18.5$ to $<25 \mathrm{~kg} / \mathrm{m}^{2}$ ), overweight $\left(\geq 25\right.$ to $<30 \mathrm{~kg} / \mathrm{m}^{2}$ ) or obese $\left(\geq 30 \mathrm{~kg} / \mathrm{m}^{2}\right)^{(27)}$. Alcohol consumption was categorised according to the classifications of the National Health and Medical Research Council (NHMRC) as non-drinker, low-risk drinker ( $\leq 14$ drinks/week), risky drinker (15-28 drinks/ week) or high-risk drinker $(28 \text { drinks/week })^{(28)}$. Self-rated health was classified as good or poor.

\section{Statistical analyses}

All analyses were completed with SAS (version 9.2; SAS Institute). Multiple logistic regression models were used to examine diabetes risk, with the risk expressed as OR with 95\% CI for each quintile of diet quality score. This approach gives results very similar to those of Cox proportional-hazards analyses with low rates of events ${ }^{(29)}$. The regression coefficients reflect the association between the incidence of diabetes and the corresponding diet quality score. Diet quality scores were categorised by quintiles, with the lowest quintile serving as the reference category. Tests for trend were carried out by entering the diet score variables into the regression models using the median score for each quintile. For each diet quality score, three models were created. Model 1 is the unadjusted estimate. Model 2 adjusted for sociodemographic and lifestyles factors and model 3 further adjusted for BMI and energy intake. Energy intake was adjusted for using the residual method described by Willett \& Stampfer ${ }^{(30)}$. All variables in these modes were treated as categorical, except energy intake, which was treated as continuous. A $P$ value $<0.05$ was considered statistically significant, and all statistical tests were two-sided.

\section{Results}

During the 6 years of follow-up, 311 incident cases of type 2 diabetes were reported. There was no statistically significant difference in mean age between women who developed type 2 diabetes and women who did not develop the disease $(t=0 \cdot 90, P=0 \cdot 37)$. The mean age of women who developed type 2 diabetes was 52.6 (SD 1.43) years compared with 52.50 (SD 1.45) years, the mean age of women who did not develop the disease. The baseline characteristics of the study sample according to the first and last quintiles of ARFS and DGI are given in Table 1. Women who scored high on the ARFS and DGI tended to have higher education and physical activity levels, better indices of self-rated health, and were less likely to be obese and to be heavy smokers and more likely to consume less alcohol and to have higher energy intakes compared with women who scored low on these diet quality scores. Women scoring high on the DGI were more likely to live in urban areas.

After adjusting for demographic characteristics, lifestyle factors and energy intake, a higher DGI score was found to be significantly and inversely associated with diabetes risk ( $P$ for trend=0.01). When comparing the quintiles, the OR for the highest $v$. the lowest quintile of DGI was found to be statistically significant (OR 0.57; 95\% CI 0.38, 0.85) (Table 2). 
Table 1. Baseline characteristics of the 8370 middle-aged women who completed the third survey of the ALSWH (Australian Longitudinal Study on Women's Health) according to the first (Q1) and fifth (Q5) quintiles of the Australian Recommended Food Score (ARFS) and Dietary Guideline Index (DGI)

(Percentages, mean values and standard deviations)

\begin{tabular}{|c|c|c|c|c|c|c|}
\hline \multirow[b]{2}{*}{ Characteristics } & \multicolumn{3}{|c|}{ ARFS } & \multicolumn{3}{|c|}{ DGI } \\
\hline & Q1 & Q5 & $P^{*}$ & Q1 & Q5 & $P^{*}$ \\
\hline \multicolumn{7}{|l|}{ Area of residence (\%) } \\
\hline Urban & 33.52 & $35 \cdot 10$ & 0.40 & 31.91 & 36.91 & 0.003 \\
\hline Large rural centre & 14.81 & $15 \cdot 38$ & & $15 \cdot 46$ & $15 \cdot 30$ & \\
\hline Small rural and remote centre & $14 \cdot 76$ & $15 \cdot 62$ & & $15 \cdot 08$ & $12 \cdot 80$ & \\
\hline Other rural and remote areas & 36.92 & 33.91 & & 37.55 & 34.98 & \\
\hline \multicolumn{7}{|l|}{ Education (\%) } \\
\hline Year 10 or equivalent & 59.09 & 36.03 & $<0.0001$ & $57 \cdot 24$ & 39.03 & $<0.0001$ \\
\hline Year 12 or equivalent & $15 \cdot 89$ & $17 \cdot 71$ & & $15 \cdot 62$ & 14.64 & \\
\hline Trade/certificate & $16 \cdot 00$ & 26.03 & & $16 \cdot 06$ & 25.08 & \\
\hline University degree & 9.01 & $20 \cdot 23$ & & 11.08 & $21 \cdot 24$ & \\
\hline \multicolumn{7}{|l|}{ Menopausal status (\%) } \\
\hline Premenopausal & 22.59 & 23.59 & 0.13 & 22.58 & 23.25 & 0.033 \\
\hline Peri-menopausal & $5 \cdot 18$ & 4.81 & & $5 \cdot 50$ & $4 \cdot 13$ & \\
\hline Postmenopausal & 0.22 & $0 \cdot 12$ & & $0 \cdot 19$ & 0.00 & \\
\hline Surgical menopausal & 23.09 & $19 \cdot 67$ & & $22 \cdot 26$ & 21.75 & \\
\hline HRT use & $15 \cdot 19$ & 14.02 & & 5.50 & $4 \cdot 13$ & \\
\hline OCP use & 33.72 & 37.79 & & $34 \cdot 27$ & 36.06 & \\
\hline \multicolumn{7}{|l|}{ BMI $(\%) \dagger$} \\
\hline Low $\left(<18.5 \mathrm{~kg} / \mathrm{m}^{2}\right)$ & 6.46 & 4.33 & $<0.0001$ & 6.07 & $3 \cdot 81$ & 0.002 \\
\hline Acceptable $\left(\geq 18.5\right.$ to $\left.<25 \mathrm{~kg} / \mathrm{m}^{2}\right)$ & 46.69 & 53.98 & & $52 \cdot 60$ & 52.53 & \\
\hline Overweight $\left(\geq 25\right.$ to $<30 \mathrm{~kg} / \mathrm{m}^{2}$ ) & 27.05 & $28 \cdot 86$ & & $25 \cdot 14$ & 28.29 & \\
\hline Obese $\left(\geq 30 \mathrm{~kg} / \mathrm{m}^{2}\right)$ & $19 \cdot 81$ & $12 \cdot 83$ & & $16 \cdot 20$ & $15 \cdot 37$ & \\
\hline \multicolumn{7}{|l|}{ Physical activity (\%) } \\
\hline Sedentary & 29.02 & $7 \cdot 39$ & $<0.0001$ & 27.46 & $9 \cdot 20$ & $<0.0001$ \\
\hline Low & 37.02 & 34.48 & & 37.09 & 37.71 & \\
\hline Moderate & $16 \cdot 01$ & 23.89 & & $15 \cdot 73$ & 24.07 & \\
\hline High & 17.95 & 34.24 & & $19 \cdot 72$ & 29.02 & \\
\hline Self-rated health as good & 85.68 & 92.90 & $<0.0001$ & 85.91 & $91 \cdot 39$ & $<0.0001$ \\
\hline \multicolumn{7}{|l|}{ Alcohol intake (\%) } \\
\hline Non-drinker & $74 \cdot 14$ & $88 \cdot 21$ & $<0.0001$ & 75.00 & $88 \cdot 61$ & $<0.0001$ \\
\hline Low-risk drinker & 19.66 & $8 \cdot 15$ & & $18 \cdot 65$ & 8.57 & \\
\hline Risky drinker & 4.73 & 3.39 & & 4.63 & $2 \cdot 50$ & \\
\hline High-risk drinker & 1.46 & 0.24 & & 1.71 & 0.31 & \\
\hline \multicolumn{7}{|l|}{ Smoking status (\%) } \\
\hline Never smoked & $51 \cdot 76$ & $57 \cdot 20$ & $<0.0001$ & $47 \cdot 17$ & $61 \cdot 75$ & $<0.0001$ \\
\hline Ex-smoker & 26.05 & 33.44 & & $25 \cdot 54$ & 31.49 & \\
\hline Smoke $<10 / d$ & 1.90 & 3.06 & & 2.92 & 1.80 & \\
\hline Smoke $10-19 / d$ & 4.84 & $2 \cdot 76$ & & $6 \cdot 24$ & 1.67 & \\
\hline Smoke $\geq 20 / d$ & 15.45 & 3.55 & & $18 \cdot 13$ & $3 \cdot 28$ & \\
\hline Energy intake $(\mathrm{kJ} / \mathrm{d}) \ddagger$ & & & $<0.0001$ & & & $<0.0001$ \\
\hline Mean & 6298 & 7395 & & 6577 & 7277 & \\
\hline SD & 2288 & 2347 & & 2290 & 2241 & \\
\hline
\end{tabular}

HRT, hormone replacement therapy; OCP, oral contraceptive pill.

${ }^{*} P$ value obtained using $\chi^{2}$ test of association.

† Weight $(\mathrm{kg}) /$ height $(\mathrm{m})^{2}$.

$\ddagger P$ value obtained using ANOVA.

Additional adjustment for BMI and energy intake did not significantly modify the observed association ( $P$ for trend $=0 \cdot 01)$. The highest quintile remained statistically significantly lower than the first quintile in terms of diabetes risk (OR 0.51; $95 \%$ CI $0.35,0.76$ ). Of all the covariates considered in the models, self-rated health and BMI had the greatest influence on the magnitude of the OR

The ARFS was not associated with type 2 diabetes risk $(P$ for trend=0.53) after adjusting for sociodemographic and lifestyle factors (Table 2). After further adjusting for BMI and energy intake, there remained no statistically significant association between the ARFS and type 2 diabetes risk ( $P$ for trend $=0 \cdot 42$ ). Comparison of diabetes risk across quintiles also remained non-statistically significant (OR for the highest $v$. the lowest quintile of ARFS 0.99; $95 \%$ CI $0.68,1.43$ ) (Table 2).

\section{Discussion}

General dietary habits within a population need to be examined for the adherence to population-specific dietary recommendations, as diet is culturally determined and dietary patterns that predict type 2 diabetes risk in different populations may not be generalisable to different populations ${ }^{(31)}$. To date in Australia, longitudinal studies evaluating the association between overall diet quality and type 2 diabetes risk 
Table 2. OR of type 2 diabetes risk by quintiles of the Australian Recommended Food Score (ARFS) and Dietary Guideline Index (DGI) among middleaged Australian women participating in the ALSWH (Australian Longitudinal Study on Women's Health) (Odds ratios and $95 \%$ confidence intervals)

\begin{tabular}{|c|c|c|c|c|c|c|c|c|c|c|}
\hline \multirow{2}{*}{$\begin{array}{l}\text { Diet quality } \\
\text { scores }\end{array}$} & Quintile 1 & \multicolumn{2}{|c|}{ Quintile 2} & \multicolumn{2}{|c|}{ Quintile 3} & \multicolumn{2}{|c|}{ Quintile 4} & \multicolumn{2}{|c|}{ Quintile 5} & \multirow{2}{*}{$\begin{array}{l}P \text { for } \\
\text { trend }\end{array}$} \\
\hline & $95 \% \mathrm{Cl}$ & OR & $95 \% \mathrm{Cl}$ & OR & $95 \% \mathrm{Cl}$ & OR & $95 \% \mathrm{Cl}$ & OR & $95 \% \mathrm{Cl}$ & \\
\hline \multicolumn{11}{|l|}{ ARFS } \\
\hline Quintile range & $6-26$ & \multicolumn{2}{|c|}{$26-32$} & \multicolumn{2}{|c|}{$32-36$} & \multicolumn{2}{|c|}{$36-41$} & \multicolumn{2}{|c|}{$41-61$} & \\
\hline Cases & 81 & \multicolumn{2}{|c|}{65} & \multicolumn{2}{|c|}{44} & \multicolumn{2}{|c|}{61} & \multicolumn{2}{|c|}{60} & \\
\hline Median & 23 & & 29 & \multicolumn{2}{|r|}{34} & \multicolumn{2}{|r|}{38} & \multicolumn{2}{|r|}{44} & \\
\hline Model $1^{*}$ & Reference & 1.43 & $1.02,1.99$ & $1 \cdot 73$ & $1.19,2.51$ & 1.36 & $0.97,1.91$ & 1.37 & $0.98,1.93$ & $0 \cdot 11$ \\
\hline Model $2 \dagger$ & Reference & 1.26 & $0.89,1.80$ & 1.33 & $0.90,1.96$ & 1.00 & $0.70,1.44$ & 0.96 & $0.69,1.39$ & 0.53 \\
\hline Model 3‡ & Reference & 1.39 & $0.96,2.02$ & 1.41 & $0.96,2.06$ & 1.03 & $0.72,1.47$ & 0.99 & $0.68,1.43$ & 0.42 \\
\hline \multicolumn{11}{|l|}{ DGI } \\
\hline Quintile range & $20-56 \cdot 5$ & \multicolumn{2}{|c|}{$56 \cdot 5-70$} & \multicolumn{2}{|c|}{$70-79$} & \multicolumn{2}{|c|}{$79-88.5$} & \multicolumn{2}{|c|}{$88.5-122.5$} & \\
\hline Cases & & & & \multirow{2}{*}{\multicolumn{2}{|c|}{$\begin{array}{l}62 \\
74.7\end{array}$}} & \multirow{2}{*}{\multicolumn{2}{|c|}{$\begin{array}{c}63 \\
83.5\end{array}$}} & \multirow{2}{*}{\multicolumn{2}{|c|}{$\begin{array}{l}39 \\
94\end{array}$}} & \\
\hline Median & $47 \cdot 25$ & \multicolumn{2}{|r|}{63.5} & & & & & & & \\
\hline Model $1^{*}$ & Reference & 0.79 & $0.57,1.12$ & 0.79 & $0.56,1.11$ & 0.77 & $0.55,1.08$ & 0.48 & $0.33,0.70$ & 0.001 \\
\hline Model $2 \dagger$ & Reference & 0.86 & $0.61,1.22$ & 0.88 & $0.62,1.26$ & 0.88 & $0.62,1.26$ & 0.57 & $0.38,0.85$ & 0.01 \\
\hline Model 3‡ & Reference & 0.79 & $0.56,1.11$ & 0.80 & $0.57,1.25$ & 0.71 & $0.49,1.03$ & 0.51 & $0.35,0.76$ & 0.01 \\
\hline
\end{tabular}

* Model 1: unadjusted.

† Model 2: adjusted for area of residence, education, physical activity, smoking status, menopausal status and self-rated health

$\ddagger$ Model 3: further adjusted for BMI and energy intake.

have been lacking. Given the lack of such research, we assessed the ability of both the ARFS and the DGI, which have been designed for use in the Australian population, to predict the risk of type 2 diabetes in a nationally representative sample of middle-aged Australian women. The findings of the present study indicated that the DGI score was inversely associated with type 2 diabetes risk during 6 years of follow-up. A previous study has shown the DGI to be a valid indicator of diet quality, reflecting intakes of key nutrients such as total fat, saturated fat, fibre, $\beta$-carotene, vitamin $\mathrm{C}$, folate, $\mathrm{Ca}$ and $\mathrm{Fe}^{(9)}$. This finding is similar to those of a previous cross-sectional study ${ }^{(12)}$, which showed inverse associations between the DGI and prevalence of type 2 diabetes among men.

A few other scoring systems and indices have emerged for the assessment of the quality of dietary patterns based on a priori defined amounts of specific food groups, as recommended by current dietary guidelines. The Alternative Healthy Eating Index, which is based on the intakes of nine components, was found to be inversely associated with type 2 diabetes risk in the Nurse's Health Study ${ }^{(5)}$. Similarly, in the Insulin Resistance Atherosclerosis Study, a measure of Dietary Approaches to Stop Hypertension (DASH) was found to be inversely associated with type 2 diabetes risk $^{(32)}$. In the Health Professionals Follow-Up Study, the Alternative Mediterranean Diet and DASH were found to be inversely associated with type 2 diabetes risk, while the Healthy Eating Index and Recommended Food Score were found to be not associated with type 2 diabetes risk $^{(6)}$. Although these tools vary slightly in terms of items included, scoring methods, assignment of items to food groups, and cut-off values, they all reflect a common dietary pattern rich in fruit and vegetables, whole grains, nuts, legumes, and fish and low in processed meat and dessert. Several mechanisms have been proposed to explain the effect of these components on diabetes pathogenesis. High intake of fibre from fruit and vegetables, whole grains, legumes and nuts improves glycaemic control by reducing or delaying the absorption of glucose, while a low glycaemic load of fruit, vegetable and milk protein minimises postprandial glucose spiking. In addition, whey has the ability to enhance the secretion of glucagon-like peptides, whereas milk proteins appear to enhance the secretion of insulinotropic amino acids and incretin hormones, which may contribute to a lower risk of type 2 diabetes $^{(33)} . \mathrm{Mg}$ from whole grains and nuts improves insulin-induced glucose uptake and oxidation ${ }^{(34)}$. Food variety was also considered in calculating those scores reflecting the number of different foods consumed within food groups over a given time period. It has been shown to be associated with better nutritional status ${ }^{(35)}$, improved physical and cognitive functions ${ }^{(36)}$, decreased morbidity ${ }^{(37-39)}$, and mortality ${ }^{(40,41)}$.

The ARFS was not statistically significantly associated with type 2 diabetes risk in middle-aged women. Lack of association between the ARFS and type 2 diabetes risk may reflect the low sensitivity of the scoring system, given that consuming a recommended food once a week adds one point to the total score, in the same way as does the consumption of the same food three or more times a week. The meat score in the ARFS includes red meat, but is not restricted to lean meat, as for the DGI. Red meat has been found to be positively associated with type 2 diabetes risk ${ }^{(42)}$. Unlike the ARFS, the DGI assesses a range of eating behaviours including energy-dense, nutrientpoor foods, which are broadly referred to as 'extra' foods in the Australian Guide to Healthy Eating ${ }^{(8)}$. A high intake of 'extra' foods has many health implications including an important role in the development of diet-related chronic diseases. The assessment of the intake of 'extra' foods when evaluating the overall diet quality is important especially among Australian adults in whom this type of food has been shown to contribute to $36 \%$ of daily energy intake ${ }^{(43)}$. As the ARFS and DGI are intended as stand-alone indicators of 
overall diet quality, we did not attempt to evaluate the individual contributions of its components to type 2 diabetes risk.

The strengths of the present study include the following: the prospective design (which minimises reverse causality); the use of a nationally representative sample of women of a similar age group (which minimises residual confounding resulting from diverse age populations); the use of a FFQ that was specifically developed and validated in the Australian population. One advantage of using the ARFS and DGI is their focus on food-based indicators, which acknowledges the complexity of dietary patterns and both nutrient and non-nutrient components of diet ${ }^{(44)}$. Nevertheless, the FFQ administered at baseline is reflective of lifelong dietary intake, which reduces the impact of possible changes in dietary behaviour due to type 2 diabetes diagnoses.

Limitations of the study should also be noted. All data were self-reported including diagnosis of type 2 diabetes, which may bias the results towards the null. Undiagnosed diabetes cases in this cohort may have been misclassified as individuals who did not develop diabetes. Misreporting of diagnosis might also be a cause of misclassification. These may have attenuated the associations observed. However, a previous study has confirmed $70 \%$ of self-reported diabetes in this cohort, which is similar to the results of previous cohort studies $^{(45,46)}$. Although potential risk factors were controlled for, information on family history of type 2 diabetes was unavailable. This may confound the observed associations, but only if diet quality is associated with family history of type 2 diabetes, as per the definition of a confounder. Because dietary intake was reported through a self-administered FFQ, misclassification of intake was another limitation. Despite the original validation study of the DQES reporting that the FFQ performed as well as other instruments used in epidemiological studies, there were considerable differences in the nutrient intakes estimated by FFQ $v$. the weighed food records at the individual level ${ }^{(23)}$. This may have affected the accuracy of the scores and attenuated the associations observed. The same validation study was restricted to only nutrient intakes. Hence, findings from the ALSWH indicated that nutrient intakes increased as diet quality score increased ${ }^{(17)}$. This indicates that the FFQ is a valid instrument to capture usual intake of the various nutrients, foods or food groups used to calculate scores for the two indices. Considering the relatively low energy intake reported by women in both scores, the possibility that the observed associations may be related to selective under-reporting cannot be excluded, in particular, among those with a higher $\mathrm{BMI}^{(47-49)}$. Under-reporting of energy intake has the potential to bias the observed results towards the null; hence, the true population effect size is likely to be stronger than that observed in the study.

In conclusion, the results of the present prospective study indicate that the DGI score, which assesses compliance with established dietary guidelines, is predictive of type 2 diabetes risk in middle-aged women. Women within the highest quintile of DGI were at approximately 50\% lower risk of type 2 diabetes compared with those in the lowest quintile, independently of potential confounders. Therefore, the DGI may be a more useful tool compared with the ARFS for assessing diet quality in future diabetes intervention studies. The validity of the ARFS is questionable, especially with regard to type 2 diabetes risk.

\section{Acknowledgements}

The authors thank all participants for their valuable contribution to the project. The ALSWH, which is conducted by researchers at the University of Newcastle and the University of Queensland, is funded by the Australian Government Department of Health and Ageing. The authors thank Professor Graham Giles of the Cancer Epidemiology Centre of Cancer Council Victoria, for permission to use the Dietary Questionnaire for Epidemiological Studies (version 2), Melbourne: Cancer Council Victoria, 1996.

A. A. is supported by a scholarship from the Ministry of Higher Education, Riyadh, Saudi Arabia.

The authors' contributions are as follows: A. A. contributed to conception and design, analysis and interpretation of the data, drafting of the manuscript and critical revision of the manuscript; E. S. contributed to statistical analyses and critical revision of the manuscript; $M$. M. contributed to conception and design and critical revision of the manuscript; W. B. contributed to acquisition of the data, analysis and interpretation of the data, obtaining funding and critical revision of the manuscript; M. L. G. contributed to conception and design and critical revision of the manuscript. All authors reviewed and approved the final manuscript.

None of the authors has any conflicts of interest to declare.

\section{References}

1. Shaw JE, Sicree RA \& Zimmet PZ (2010) Global estimates of the prevalence of diabetes for 2010 and 2030. Diabetes Res Clin Pract 87, 4-14

2. Hu FB, Manson JE, Stampfer MJ, et al. (2001) Diet, lifestyle, and the risk of type 2 diabetes mellitus in women. $N$ Engl $J$ Med 345, 790-797.

3. Mozaffarian D, Kamineni A, Carnethon M, et al. (2009) Lifestyle risk factors and new-onset diabetes mellitus in older adults: The Cardiovascular Health Study. Arch Intern Med 169, 798-807.

4. Alhazmi A, Stojanovski E, McEvoy M, et al. (2014) The association between dietary patterns and type 2 diabetes: a systematic review and meta-analysis of cohort studies. J Hum Nutr Diet 27, 251-260.

5. Fung TT, McCullough M, van Dam RM, et al. (2007) A prospective study of overall diet quality and risk of type 2 diabetes in women. Diabetes Care 30, 1753-1757.

6. de Koning L, Chiuve SE, Fung TT, et al. (2011) Diet-quality scores and the risk of type 2 diabetes in men. Diabetes Care 34, 1150-1156.

7. National Health and Medical Research Council (2003) Dietary Guidelines for Australian Adults. Canberra, ACT: Australian Government Publishing Service.

8. Smith A, Kellet E \& Schmerlaib Y (1998) The Australian Guide to Healthy Eating. Canberra, ACT: Commonwealth Department of Health and Family Services.

9. McNaughton SA, Ball K, Crawford D, et al. (2008) An index of diet and eating patterns is a valid measure of diet quality in an australian population. J Nutr 138, 86-93. 
10. Kennedy ET, Ohls J, Carlson S, et al. (1995) The Healthy Eating Index: design and applications. J Am Diet Assoc 95, $1103-1108$

11. Haines PS, Siega-Riz AM \& Popkin BM (1999) The Diet Quality Index revised: a measurement instrument for populations. J Am Diet Assoc 99, 697-704.

12. McNaughton SA, Dunstan DW, Ball K, et al. (2009) Dietary quality is associated with diabetes and cardio-metabolic risk factors. J Nutr 139, 734-742.

13. Arabshahi S, van der Pols JC, Williams GM, et al. (2011) Diet quality and change in anthropometric measures: 15-year longitudinal study in Australian adults. Br J Nutr 107, $1376-1385$

14. Arabshahi S, Lahmann PH, Williams GM, et al. (2011) Longitudinal change in diet quality in Australian adults varies by demographic, socio-economic, and lifestyle characteristics. J Nutr 141, 1871-1879.

15. Collins CE, Young AF \& Hodge A (2008) Diet quality is associated with higher nutrient intake and self-rated health in mid-aged women. J Am Coll Nutr 27, 146-157.

16. Kant AK \& Thompson FE (1997) Measures of overall diet quality from a food frequency questionnaire: National Health Interview Survey, 1992. Nutr Res 17, 1443-1456.

17. Hure A, Young A, Smith R, et al. (2009) Diet and pregnancy status in Australian women. Public Health Nutr 12, 853-861.

18. Collins CE, Patterson A \& Fitzgerald D (2011) Higher diet quality does not predict lower medicare costs but does predict number of claims in mid-aged Australian women. Nutrients 3, 40-48.

19. Lee C, Dobson AJ, Brown WJ, et al. (2005) Cohort Profile: The Australian Longitudinal Study on Women's Health. Int J Epidemiol 34, 987-991.

20. Alhazmi A, Stojanovski E, McEvoy M, et al. (2013) Macronutrient intake and type 2 diabetes risk in middle-aged Australian women. Results from the Australian Longitudinal Study on Women's Health. Public Health Nutr 17, $1587-1594$

21. Ireland PJD, Giles G, O'Dea K, et al. (1994) Development of the Melbourne FFQ: a food frequency questionnaire for use in an Australian prospective study involving an ethnically diverse cohort. Asia Pac J Clin Nutr 3, 19-31.

22. National Food Authority (1995) Nutrient Data Table for Use in Australia. Canberra, ACT: National Food Authority

23. Hodge A, Patterson AJ, Brown WJ, et al. (2000) The Anti Cancer Council of Victoria FFQ: relative validity of nutrient intakes compared with weighed food records in young to middle-aged women in a study of iron supplementation. Aust N Z J Public Health 24, 576-583.

24. Lowe J, Byles J, Dolja-Gore X, et al. (2010) Does systematically organized care improve outcomes for women with diabetes? J Eval Clin Pract 16, 887-894.

25. Australian Institute of Health and Welfare (2004) Rural, Regional and Remote Health: A Guide to Remoteness Classifications. Canberra, ACT: AIHW.

26. Armstrong T, Bauman A \& Davis J (2000) Physical Activity Patterns of Australian Children. Canberra, ACT: Australian Institute of Health and Welfare.

27. World Health Organization (2000) Obesity: Preventing and Managing the Global Epidemic. Geneva: WHO.

28. National Health and Medical Research Council (2001) Australian Alcohol Guidelines: Health Risks and Benefits. Canberra, ACT: Commonwealth of Australia.

29. D'Agostino RB, Lee ML, Belanger AJ, et al. (1990) Relation of pooled logistic regression to time dependent Cox regression analysis: the Framingham Heart Study. Stat Med 9, $1501-1515$

30. Willett W \& Stampfer MJ (1986) Total energy intake: implications for epidemiologic analyses. Am J Epidemiol $\mathbf{1 2 4}$ $17-27$.

31. Imamura F, Lichtenstein AH, Dallal GE, et al. (2009) Generalizability of dietary patterns associated with incidence of type 2 diabetes mellitus. Am J Clin Nutr 90, 1075-1083.

32. Liese AD, Nichols M, Sun X, et al. (2009) Adherence to the DASH diet is inversely associated with incidence of type 2 diabetes: the insulin resistance atherosclerosis study. Diabetes Care 32, 1434-1436.

33. King JC (2005) The milk debate. Arch Intern Med 165 975-976.

34. Larsson SC \& Wolk A (2007) Magnesium intake and risk of type 2 diabetes: a meta-analysis. I Intern Med 262, 208-214.

35. Bernstein MA, Tucker KL, Ryan ND, et al. (2002) Higher dietary variety is associated with better nutritional status in frail elderly people. I Am Diet Assoc 102, 1096-1104.

36. Clausen T, Charlton KE, Gobotswang KSM, et al. (2005) Predictors of food variety and dietary diversity among older persons in Botswana. Nutrition 21, 86-95.

37. Fernandez E, D'Avanzo B, Negri E, et al. (1996) Diet diversity and the risk of colorectal cancer in northern Italy. Cancer Epidemiol Biomarkers Prev 5, 433-436.

38. La Vecchia C, Munoz SE, Braga C, et al. (1997) Diet diversity and gastric cancer. Int J Cancer 72, 255-257.

39. Fernandez E, Negri E, La Vecchia C, et al. (2000) Diet diversity and colorectal cancer. Prev Med 31, 11-14.

40. Kant AK, Schatzkin A, Harris TB, et al. (1993) Dietary diversity and subsequent mortality in the First National Health and Nutrition Examination Survey Epidemiologic Follow-up Study. Am J Clin Nutr 57, 434-440.

41. Kant AK, Schatzkin A \& Ziegler RG (1995) Dietary diversity and subsequent cause-specific mortality in the NHANES I epidemiologic follow-up study. J Am Coll Nutr 14, 233-238.

42. Pan A, Sun Q, Bernstein AM, et al. (2011) Red meat consumption and risk of type 2 diabetes: 3 cohorts of US adults and an updated meta-analysis. Am J Clin Nutr 94, 1088-1096.

43. Rangan AM, Schindeler S, Hector DJ, et al. (2008) Consumption of 'extra' foods by Australian adults: types, quantities and contribution to energy and nutrient intakes. Eur J Clin Nutr 63, 865-871.

44. Kant AK (1996) Indexes of overall diet quality: a review. J Am Diet Assoc 96, 785-791.

45. Meyer KA, Kushi LH, Jacobs DR, et al. (2000) Carbohydrates, dietary fiber, and incident type 2 diabetes in older women Am J Clin Nutr 71, 921-930.

46. Sluijs I, Beulens JW, van der A DL, et al. (2010) Dietary intake of total, animal, and vegetable protein and risk of type 2 diabetes in the European Prospective Investigation into Cancer and Nutrition (EPIC)-NL study. Diabetes Care 33, 43-48.

47. Heitmann BL \& Lissner L (1995) Dietary underreporting by obese individuals - is it specific or non-specific? BMJ $\mathbf{3 1 1}$ 986-989.

48. Rennie KL, Johnson L \& Jebb SA (2005) Behavioural determinants of obesity. Best Pract Res Clin Endocrinol Metab 19, 343-358.

49. Rodriguez G \& Moreno LA (2006) Is dietary intake able to explain differences in body fatness in children and adolescents? Nutr Metab Cardiovasc Dis 16, 294-301. 